International Journal of Computational Intelligence Research

ISSN 0973-1873 Volume 9, Number 2 (2013), pp. 79-88

(C) Research India Publications

https://dx.doi.org/10.37622/IJCIR/9.2.2013.79-88

\title{
A Review of Modern HCI Challenges for Betterment of Differently Abled People
}

\author{
Papri Ghosh $^{1}$, Tejbanta Singh Chingtham², \\ Mrinal Kanti Ghose ${ }^{3}$ and Ritam Dutta ${ }^{4}$ \\ ${ }^{1}$ Asst. Prof., Dept. of CSE, SIEM, WBUT, \\ ${ }^{2}$ Prof., Dept. of CSE, SMIT, SMU, \\ ${ }^{3}$ Prof., Dean $R \&$ D, Dept. of CSE, SMIT, SMU, \\ ${ }^{4}$ Asst. Prof., Dept. of ECE, SIEM, WBUT,
}

\begin{abstract}
In todays' world, the latest cognitive technologies available in markets are not comprehensible to all the individuals. However some technologies are available that helps all the individuals to be more interactive with others in the society thereby reducing the technological divide. Some of the products are there like audio navigation system for blind people or video navigation system for deaf people or voice command wheel chair for physical disabled people etc. are enabling them to live better without any help of any other fellow human being. Most of the cases consider either lesser interactive device with less accessibility or highly cost effective tools have been used. There is lacks of cost effective intelligent interactive systems for an individual.

This study proposes to develop a holistic approach using Human Computer Interaction to design an interactive system that minimize the barrier between the human's cognitive model of what they want to accomplish and the computer's understanding of the user's task by integrating techniques or applications to enable an individual to use the advanced technologies in more interactive manner. It is also planned to develop a prototype with minimal complexities and by retaining and enhancing the required features, thereby providing a supportive system that can meet the need of its deliberate users.
\end{abstract}

Index Terms-Cognitive Technology, Human Computer Interaction, Differently Abled People. 


\section{INTRODUCTION}

In the advent of meteorological growth and advancement of computer technologies, things are no more as simple as it was earlier. With the increase in high efficiency of speed, accuracy and user friendliness, the advent of technology has become more complex. Effort and edification have become inseparably to the individuals' everyday lives in our current society and they are the main mediators for a successful social subsistence.

Human-Computer Interaction is a discipline concerned with the design, evaluation and implementation of interactive computing systems for human use and with the study of major phenomena surrounding them [1]. In other words, Human-computer Interaction is a discipline concerned with the study, design, construction and implementation of human-centric interactive computer systems [2]. The term was popularized by Card, Moran, and Newell in their book, "The Psychology of HumanComputer Interaction", 1983 although the authors first used the term in 1980 [3]. Utilizing computers had always been the question of interfacing. The methods by which human has been interacting with computers has come a long way. The journey still continues with new designs of technologies and as systems becomes more and more sophisticated each day, the research in this area has been growing at a very fast pace in the last few decades. The growth in the field of Human-Computer Interaction (HCI) has not only been in quality of interaction, it has also experienced different branches of it in its history [10].

Sometimes called as Man-Machine Interaction or Interfacing, concept of HumanComputer Interaction /Interfacing (HCI) was automatically represented with the emergence of computer, or more generic machine. The reason, in fact, is clear: most sophisticated machines are worthless unless they can be used properly by men. This basic argument simply presents the main terms that should be considered in the design of HCI: functionality and usability. Why a system is actually designed can ultimately be defined by what the system can do i.e. how the functions of a system can help towards the achievement of the purpose of the system. Functionality of a system is defined by the set of actions or services that it provides to its users. However, the value of functionality is visible only when it becomes possible to be efficiently utilized by the user. Usability of a system with a certain functionality is the range and degree by which the system can be used efficiently and adequately to accomplish certain goals for certain users. The actual effectiveness of a system is achieved when there is a proper balance between the functionality and usability of a system [4].

Having these concepts in mind and considering that the terms computer, machine and system are often used interchangeably in this context, HCI is a design that should produce a fit between the user, the machine and the required services in order to achieve a certain performance both in quality and optimality of the services. Determining what makes a certain HCI design good is mostly subjective and context dependant. The available technology could also affect how different types of HCI are designed for the same purpose. One example is using commands, menus, graphical user interfaces (GUI), or virtual reality to access functionalities of any given computer. The advances made in last decade in HCI have almost made it impossible to realize which concept is invention and which is and can be genuine [7]. The thrust 
in research and the constant twists in marketing cause the new technology to become available to everyone in no time. However, not all existing technologies are accessible and/or affordable by public.

Research in this area is voluminous; a complete branch of computer science is devoted to this topic, with recommendations for the proper design of menus, icons, forms, as well as data display and entry screens. The internet browser is an example of Human Computer Interface where interfaces such as - the buttons and menus have been designed to make it easy for user to access the web. A Computer Mouse, Touch Screen, A program on Mac or Windows machine that includes a trashcan, icons of disk drives, folders, and pull-down menus are all examples of advances in humancomputer interface design which were designed to make it easier to accomplish things with a computer [15]. Many of these recommendations concern the design of computer interfaces like Windows or the Mac Finder or how to make programs easier to use. Some of these recommendations are not so relevant to web design. Still, it is an important area of research, and some of the recommendations relate to any kind of communication between user and computer.

The Human Computer Interaction design seeks to discover the most efficient way to design understandable electronic messages. It involves Study, Planning and Design of the interaction between users and computers. Three major components of Human Computer Interaction (HCI) are:

- Perception

- Interaction \&

- Accessibility

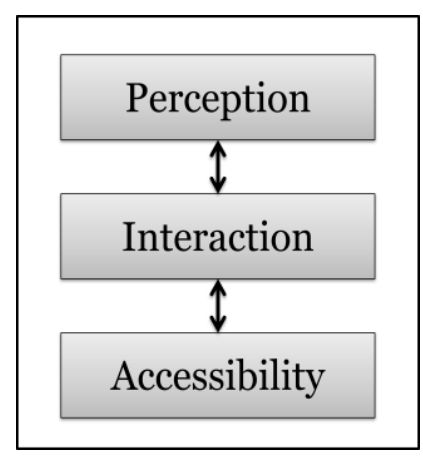

Fig.1 Relationship between Perception, Interaction and Accessibility

\section{Perception:}

Perception is the mechanism to acquire knowledge about the environment either by sensory organs in living beings or some form of artificial sensors. Artificial perception is achieved by taking measurements using various sensors and then extracting meaningful information from those measurements.

In human, it can be used to determine the level and capacity of understanding of an individual. Decisions taken based on the information of the sensory organs and the capacity of understanding of the collected information of the environment in 
conjunction with the brain is called Cognition. Hence the level of cognition can be said to be directly proportional to the level of perception. Artificial aid/sensor is deployed for used by human with various grades of disabilities to assist or enhance the cognition.

\section{Interaction:}

The interaction is the sense of response either naturally or by any technological aid. It comprises of decision making and identifying the component that should be deployed to ensure a correct response is generated after processing the perceived information. Interaction in the early days of computation was referred to as "interface" as it allows a text-mode only operating system to explore the resources of the computer through a mechanism called GUI. However with the advent of technology and the introduction of HCI, the system has evolved to such an extent that the interface no longer remains an ordinary interface rather a technique by which various communication methods of humans are also recognized to exploit the resources of a computer. And so much so that the term Human Computer Interface is renamed as Human Computer Interaction. The Interaction phase is the core area of HCI where any technological intervention can take place, as the level of sensing of any form of sensor will remain the same. Translating all forms of information to appropriate meaning can be done using the decision making phase of the Interaction system.

\section{Accessibility:}

Accessibility determines the ease and convenience of a chosen component to perform a requested task. If a wrong component of a system is chosen by the decision making phase of the Interaction phase than the level of accessibility will be drastically reduced. Similarly a faulty but correct part may not produce the desired result. Hence accessibility determines the final output of a system. In the absence of a correct component/part of a system to produce the desired result, the Interface module may be informed a priory and a combination of other component/parts may be sought to seek closest to the desired result. For example, in human, a person with hearing disability may be supported with hearing aid to enable him to understand the sound/ speech that can be heard in his surroundings. On a similar note, the basic function of HCI is to ensure that the interface part takes appropriate decision to augment the accessibility options of a system.

\section{Literature Survey}

D. Chowdhuri et al. proposed a Virtual Classroom System for deaf people in 2012, which provides a scope of learning through bilingual method [16]. The proposed system provides many learning facilities for its users and supports many tasks which would help the users to learn and also provides security to the data by providing access to authorized people only. An algorithm has been designed for truthful authentication for users. The system also represents a mixing of the interactive \& public tools jointly with conversion to sign languages which increases the usability and interactivity within virtual learning environment for disabled users. The system 
could be presented in an additional interactive way with online applications. SMS alert might be programmed for better communication with the registered users.

\section{Literature Gap}

The system could be presented in an additional interactive way with online applications. The SMS alert might be programmed for better communication with the registered users.

M. G. Grif et al., in 2001, considered a computer sign language translation technology for deaf people [13]. The Russian text analysis stage, including prior semantic and semantic interpretation within the framework of developing a program for Russian text computer sign language translator into colloquial Russian sign language is given. Some global language with any other language conversion technique could be considered to apply the system around the world.

\section{Literature Gap}

Global Language with any other language conversion technique could be considered.

Limited research has been done on creating accessible Web based systems for individuals with cognitive and learning disabilities. The well understood impairments for a visually impaired person need a system with web accessibility. G. D. Fryia et al. in 2009 described current human-computer interaction research for cognitive and/or learning disabled individuals [11]. They also outlined several human-computer interaction challenges that arise during designing web-based systems for them. Furthermore, a sample e-Learning system was designed for simplifying many difficult steps used in the online systems for these individuals. Most frequently-cited design which was recommended with graphics, icons, and symbols in addition to text was incorporated in the interface. The interface could be designed with more number of options or icons and menus available for the CLD's may be increased with more visual effects for the higher level of interaction.

\section{Literature Gap}

The interface could be designed with more number of options or icons and menus available for the CLD's may be increased with more visual effects for the higher level of interaction.

To assure the equal access of information, knowledge, education and employment, the improvement of the educational procedures and techniques is very important. For these A. Drigus et al. on 2006 presents a multi-purpose e-environment for educative and informative services in the domain of engineering education for students with visual impairments [6]. To produce a user friendly and easily navigable environment for this nontraditional group of students special "Assistive Technology" was used and the principles of "Design for All" and "Universal Accessibility" were followed. For additional interaction for all the impaired users, the necessary software can be installed by default for minimal complexities.

\section{Literature Gap}

For additional interaction for all the impaired users, the necessary software can be 
installed by default for minimal complexities.

S. Hemachandra et al. in 2011 proposed a tour taking robot that autonomously follows a human tour guide in a society acceptable way and segment the acquire freespaced map into spaces that are meaningful to humans and infer a label for each space [5]. The system involved simultaneous localization and mapping (SLAM), Human Computer Interaction (HCI), person following, spoken dialog measurement, map segmentation and Human-Augmented Mapping (HUM). An improved system having the capacity of acceptance of complex robotics systems in everyday human environments and improvement of the quality of life of people in assisted-living environments can be designed.

\section{Literature Gap}

An improved system having the capacity of acceptance of complex robotics systems in everyday human environments and improvement of the quality of life of people in assisted-living environments can be designed.

According to T. Chambel et al., 2007, an idea was presented to teach Human Computer Interaction (HCI) to blind students [8]. They took a challenge to teach two blind students with other normal students and described their experiences with some possible solutions. The accessibility awareness among teachers about their diverse learners and how to go about helping them to learn better is important to increase.

\section{Literature Gap}

The accessibility awareness among teachers about their diverse learners and how to go about helping them to learn better is important to increase.

R. B. Hink et al. in 2010, presented a design of a Graphical User Interface (GUI) depending upon behavioral characteristics of a blind person [12]. The environment aims to construct based on interviews and questionnaires conducted with various blind people. Further usability evaluation is being conducted and will provide feedback from live recording of usage and questionnaires as well as interviews to make the interaction level to be more efficient.

\section{Literature Gap}

More usability evaluation can be conducted for high interaction level.

Most of the environments of Human Computer Interaction (HCI) are deployed for deep understanding of user characteristics methodologies for building interface to software applications. J. C. Muzio et al. in 2001 designed software to be used in the Traumatic Brain Injury Program at a local hospital to assist the cognitive rehabilitation of patients with brain injuries and gathered feedback from therapists and patients in the hospital environment to refine our product [14]. The required a considerable amount of work on the therapists' behalf to complete this process, from the beginning consultation and extensive questionnaire to the discussions during the beta testing phase, and their total involvement in the development of the enhancements. 


\section{Literature Gap}

An extensive questionnaire to the discussions during the beta testing phase can be done for increasing the interaction level.

F.Karray et al. in 2008 has given a description of a brief overview on Human Computer Interaction (HCI) with its definition and terminology, survey for existing technologies, recent advances in the field and common architectures used in the design of HCI systems which includes unimodal and multimodal configurations, and finally the applications of HCI [10]. It is analyzed that enormous amount of attention has been paid to better designs of HCI. The new direction of research is to replace common regular methods of interaction with intelligent, adaptive, multimodal, natural methods. With the elaborative description of latest used application design it is analyzed that a lots of further research work are still left on existing technologies to achieve some other goals.

\section{Literature Gap}

With the elaborative description of latest used application design it is analyzed that a lots of further research work are still left on existing technologies to achieve some other goals.

V. Hinze-Hare in 2007 presented three Human Computer Interaction (HCI) models and structure viz., Computer Supported Collaborative Working (CSCW), Computer Supported Collaborative Learning (CSCL) \& Computer Supported Collaborative Research (CSCR) are compared to find the most popular model [9]. A Use Authors' citation frequency as a weighting factor has been calculated and analyzed. Since 1980 there has been tremendous development in the field of HCI and more research is still being carried out to enhance the study of HCI. Hence lots of scope.

\section{Literature Gap}

Since 1980 there has been tremendous development in the field of HCI and more research is still being carried out to enhance the study of HCI. Hence lots of scope.

\section{Highlights of Literature Gap}

Human Computer Interaction (HCI) has established its domain in the field of computer science and has successfully evolved as a new research paradigm. However, as on date there are lots of scopes, in spite of present, work are there towards the application of Human Computer Interaction (HCI). After performing an adequate survey in various disabilities of human being, the following points enlighten the highlights of research gap:

- Full functional interactive systems with online applications, more visual effects, global language with any other language conversion design and elaborative design for the users are very necessary. None of the research proposed the full functional interactive system for disable people.

- Some of the research didn't consider global language which can be converted into another language and applicable around the world.

- The proper algorithm for HCI design for Interaction Level is missing. 
- The interaction level of the system with an individual can be more elaborative after accepting his/her perception level.

- No algorithm has been given for the situation if Interactive Level is Nil.

\section{Conclusion}

This paper depicts the collective approaches in Human Computer Interactions (HCI) Design for betterment of Differently Abled People (DAP). The disabilities of human being are of many types, whose proper solutions with methodologies have been proposed by many researchers. The detail survey of several literatures has been done thoroughly, reviewed and the limitations in respective literature have been found out for finding the ultimate solutions of above mentioned disabilities.

\section{Future Scope}

In the modern era the Human Computer Interaction (HCI) Design face lot of challenges, as there are increasing number of disabilities handicap people now-a-days. Several number of Human Computer Interaction (HCI) Methodologies can be used to design in a very efficient way by applying proper algorithms, so that implementing those design any solution for the disabilities can be reduced to some extent. The proposed system must be cost effective so that it can help the disable human being with any class.

\section{References}

[1] "ACM SIGCHI Curricula for Human-Computer Interaction", Definition of HCI, [Available: http://old.sigchi.org/cdg/cdg2.html]

[2] "Human Computer Interaction (HCI)", [Available: http://www.webopedia.com/TERM/H/HCI.html]

[3] "Human-computer Interaction", [Available: https://en.wikipedia.org/wiki/Human-computer_interaction]

[4] Brad A. Myers. "A Brief History of Human Computer Interaction Technology." ACM interactions. Vol. 5, no. 2, March, 1998. pp. 44-54.

[5] S. Hemachandra, T. Kollar, N. Roy and S. Teller, "Following and Interpreting Narrated Guided Tours". In the proceedings of ICRA 2011 [Available : http://groups.csail.mit.edu/rrg/papers/sachi_icra11.pdf]

[6] A. Drigas, L. Koukianakis, Y. Papagerasimou. "An E-Learning Environment for Nontraditional Students with Sight Disabilities". 36th ASEE/IEEE Frontiers in Education Conference S1J-23, October 28 - 31, 2006, San Diego, CA.

[7] M. Fetaji, S. Loskoska, B. Fetaji, M. Ebibi. "Investing Human Computer Interaction Issues in Designing Efficient Virtual Learning Envioronment". BCI 2007, Sofia, Bulgaria. 
[8] T. Chambel, P. Antunes, C. Duarte, L. Carriço, N. Guimarães. "Teaching Human-Computer Interaction to Blind Students”. HCI Educators 2007, Aveiro, Portugal.

[9] V. Hinze-Hoare. "Review and Analysis of Human Computer Interaction (HCI) Principles". Southampton University. July 2007. pp. 1-13.

[10] F. Karray, M. Alemzadeh, J. A. Saleh and M. N. Arab. "Human-Computer Interaction: Overview on State of the Art". In the proceedings of IJSSIS, Vol. 1, No. 1, March 2008, pp. 137-159.

[11] G. D. Fryia, R. W. Smolikova, M. P. Wachowiak. "Web Accessibility in the Development of an e-Learning System for Individuals with Cognitive and Learning Disabilities". 1st Int. Conf. on Networked Digital Technologies, on 28-31 July 2009. pp. $153-158$.

[12] R. B. Hink, A. A. Suarez. "Basic Human Computer Interface for the Blind". LACCEI'2010, Vol.8, June 2010.

[13] M.G. Grif, "Development of computer sign language translation technology for deaf people", Strategic Technology (IFOST), 6th International Forum. 22-24 Aug. 2011. Volume: 2. pp. $674-677$.

[14] J. C. Muzio and M. Serra, "HCI Challenges In Designing for Users with Disabilities". In the proceedings of ICHCI, Vol. 1, August 2011, pp. 46-50.

[15] K. R. A. Kumar, S. Ravi, S. K. Srivatsa. "Effective e-learning approach for Students with Learning Disabilities". IJSER, Vol. 2, Issue 11, Nov 2011, ISSN 2229-5518.

[16] D. Chowdhuri, N. Pard, A. Maity. "Virtual classroom for Deaf people", IEEE Transaction on Innovative Practices and Future Trends (AICERA), 19-21 July 2012, pp 1-3. 


\section{Authors}

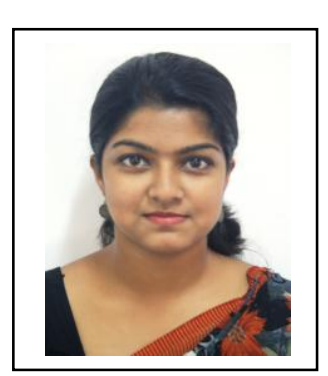

\section{Papri Ghosh}

She did her B.Tech. in Information Technology from SIT, West Bengal University of Technology in 2008 \& M. Tech. in Computer Science \& Engineering from NITTTR Kolkata, West Bengal University of Technology in 2010. Presently she is working as Assistant Professor in Surendra Institute of Engineering \& management, Siliguri in the Dept. of C.S.E and also holding key positions in the institute. Her current research focuses on Artificial Intelligence, e-Learning, Expert System, Secured Online Biometric Authentication System. She is a member of UACEE, AIRCC and IACSIT. She has published book and several research papers in International \& National Journals and Conferences.

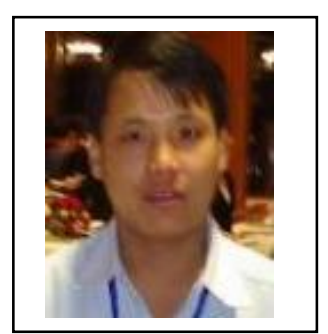

\section{Tejbanta Singh Chingtham}

He is associated with Sikkim Manipal Institute of Technology since August 2001 and is working as a Professor in the Dept. of Computer Science \& Engineering. He received his B.Tech in Computer Science \& Engineering from Bharathiar University, Coimbatore, India. He did his M.Tech from IIT Guwahati in Computer Science \& Engineering \& $\mathrm{Ph.D}$. from Birla Institute of Technology, Mesra, India. $\mathrm{He}$ is a member of IEEE, IAENG, and IACSIT. He has served as technical committee members of various Journals \& Conferences.

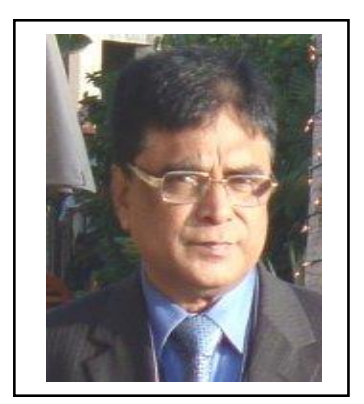

\section{Mrinal Kanti Ghose}

$\mathrm{He}$ is a former senior scientist in Regional Remote Sensing Service Centre (ISRO/DOS), Kharagpur, India. Prior to this assignment he was working as a Senior Scientist in the System Reliability Group, Vikram Sarabhai Space Centre (ISR), Trivundrum, India. He did his Master's and Ph.D. in Statistics respectively. He has almost 36 years of research and teaching experience. Currently he is Dean R \& D in Sikkim Manipal University and Professor (H.O.D.) in Dept. of CSE, SMIT, Sikkim. His research areas are Geo Informatics, Networking \& Information Security, Data Mining, Software Engineering, Robotics, System Reliability and Quality Assurance. He is a life member of ISRS, IIRS Dehradun and NIQR Trivundrum.

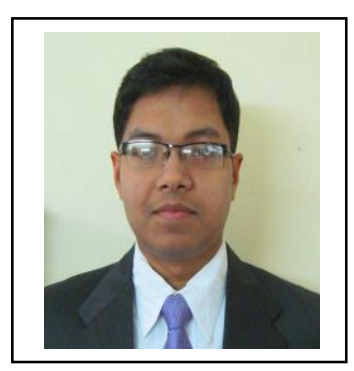

\section{Ritam Dutta}

He earned his B.Tech. in Electrical \& Electronics Engineering from SMIT, Sikkim Manipal University in 2007 \& M. Tech. in Electronics \& Communication Engineering (VLSI Design) from SRM University, Chennai in 2009. Presently he is working as Assistant Professor in Surendra Institute of Engineering \& Management, Siliguri in the Dept. of E.C.E. He has 4 years of teaching experience. His current research focuses on Secured online Biometric Authentication System, VLSI Design, E-Learning. He has published a book on bio-metrics and several research papers in International \& National Conferences and Journals of repute. He is also an associative member of several International Associations such as ISTE, IAENG, UACEE, IACSIT etc. 